\title{
Nuclear Magnetic Shieldings of Stacked Aromatic and Antiaromatic Molecules
}

\section{Sundholm, Dage}

2017-05

Sundholm , D , Rauhalahti , M , Özcan , N , Mera-Adasme , R , Kussmann , J , Luenser , A \& Ochsenfeld , C 2017 , ' Nuclear Magnetic Shieldings of Stacked Aromatic and Antiaromatic Molecules ' , Journal of Chemical Theory and Computation, vol. 13 , no. 5 , pp. 1952-1962 . https://doi.org/10.1021/acs.jctc.6b01250

http://hdl.handle.net/10138/309509

https://doi.org/10.1021/acs.jctc.6b01250

unspecified

acceptedVersion

Downloaded from Helda, University of Helsinki institutional repository.

This is an electronic reprint of the original article.

This reprint may differ from the original in pagination and typographic detail.

Please cite the original version. 


\title{
Nuclear magnetic shieldings of stacked aromatic and antiaromatic molecules
}

\author{
Dage Sundholm, ${ }^{*, \dagger}$ Markus Rauhalahti, ${ }^{\dagger}$ Nergiz Özcan, ${ }^{\dagger}$ Raúl Mera-Adasme, ${ }^{\ddagger}$ \\ Jörg Kussmann," Arne Luenser, " and Christian Ochsenfeld*, \\ University of Helsinki, Department of Chemistry, P.O. Box 55 (A.I. Virtanens plats 1), \\ FIN-00014 University of Helsinki, Finland, Departamento de Ciencias del Ambiente, \\ Universidad de Santiago de Chile (USACH), Av. Libertador Bernardo O'Higgins 3363. \\ Estación Central, Chile, and Chair of Theoretical Chemistry, Department of Chemistry, \\ University of Munich (LMU), München D-81377, Germany
}

E-mail: Dage.Sundholm@helsinki.fi; christian.ochsenfeld@cup.uni-muenchen.de

\footnotetext{
${ }^{*}$ To whom correspondence should be addressed

${ }^{\dagger}$ University of Helsinki, Department of Chemistry, P.O. Box 55 (A.I. Virtanens plats 1), FIN-00014 University of Helsinki, Finland

${ }^{\ddagger}$ Departamento de Ciencias del Ambiente, Universidad de Santiago de Chile (USACH), Av. Libertador Bernardo O'Higgins 3363. Estación Central, Chile

"Chair of Theoretical Chemistry, Department of Chemistry, University of Munich (LMU), München D81377, Germany
} 


\begin{abstract}
Nuclear magnetic shieldings have been calculated at the density functional theory (DFT) level for stacks of benzene, hexadehydro[12] annulene, dodecadehydro[18]annulene and hexabenzocoronene. The magnetic shieldings due to the ring currents in the adjacent molecules have been estimated by calculating nucleus independent molecular shieldings for the monomer in the atomic positions of neighbor molecules. The calculations show that the independent shielding model works reasonable well for the ${ }^{1} \mathrm{H}$ NMR shieldings of benzene and hexadehydro[12]annulene, whereas for the larger molecules and for the ${ }^{13} \mathrm{C}$ NMR shieldings the interaction between the molecules leads to shielding effects that are at least of the same size as the ring current contributions from the adjacent molecules. A better agreement is obtained when the nearest neighbors are also considered at full quantum mechanical (QM) level. The calculations suggest that the nearest solvent molecules must be included in the quantum mechanical system, at least when estimating solvent shifts at the molecular mechanics (MM) level. Current density calculations show that the stacking does not significantly affect the ring current strengths of the individual molecules, whereas the shape of the ring current for a single molecule differs from that of the stacked molecules.
\end{abstract}

\title{
1 Introduction
}

Environmental effects are often taken into account by performing calculations using a combined quantum and molecular mechanical (QM/MM) treatment. ${ }^{1}$ In $\mathrm{QM} / \mathrm{MM}$ calculations, the investigated molecule is considered at the QM level, whereas the electrostatic interactions with the surrounding molecules are treated at the classical level of theory using point charges. Realistic solvent contributions can be obtained as the average value by performing molecular dynamic (MD) simulations and recalculating the property under consideration for

many solvent configurations taken from the MD simulation. ${ }^{2,3}$ It might though be necessary to include the solvent molecules nearest to the solute in the QM system, especially when the 
interactions between the solvent and the solute are strong. An accurate but computationally expensive approach is to completely omit the MM part. Instead, the size of the quantum mechanical system is enlarged until the studied property is not significantly affected by increasing the system size. ${ }^{4}$ Also in the full QM approach, the investigated property has to be sampled over a large number of solvent configurations rendering the calculations computationally expensive. Frozen-density embedding (FDE) calculations have also been employed in calculations of solvent effects. ${ }^{5,6}$ The embedding potential of the FDE calculations is deduced from a separate calculation using the average electron density of many configurations of the solvent molecules, whereas the QM calculation on the solute is performed only once. ${ }^{7}$ The more traditional approach to calculate solvent effects is the use of continuum models such as the conductor-like screening (COSMO) and the polarizable continuum models (PCM). ${ }^{8-11}$ The common denominator for the approximate approaches is that they may be well aimed for considering electrostatic effects as the environment is simulated with point charges or using a continuous dielectric medium, whereas they may be less appropriate for studies of magnetic properties, ${ }^{12,13}$ because the magnetically induced current densities in the solvent molecules are not taken into account. In molecular solids, the ring currents of the individual aromatic rings significantly shift the peaks in the measured NMR spectra. ${ }^{14-21}$ Current densities of neighboring molecules might bring significant contributions to magnetic properties, even from molecules that are far away from the molecule under consideration. ${ }^{22}$ Thus, a series of cluster calculations at the QM level are so far the only means to consider the most important contributions to the environmental effects on magnetic properties, even though several attempts with QM/MM approaches have been made. ${ }^{23-28}$ Linear scaling and nucleus selected calculations open the avenue towards computational studies of NMR chemical shifts for large molecular systems. ${ }^{29-32}$

For polar solvents, the solvent shift originating from the magnetically induced current densities of the solvent molecules is usually smaller than the contributions due to the electrostatic moments of the solvent molecules. Hydrogen bonding and van der Waals interactions 
between solvent molecules and the solute as well as structural relaxation of the solute also affect NMR chemical shifts. Electrostatic effects are less pronounced for nonpolar molecules, whereas external magnetic fields induce strong ring currents in aromatic molecules resulting in an additional magnetic field contribution that significantly affects the NMR chemical shifts. Since aromatic solvent molecules surrounding the solute contribute to the observed magnetic properties of the solute, ${ }^{26,33,34}$ some kind of cluster model has to be employed when aiming at very accurate calculations of solvent effects on magnetic properties. Calculations on crystalline benzene have shown that the closest neighbor molecules have to be included in the QM treatment together with the solute, whereas the contributions from more distant solvent molecules may be estimated by performing calculations of nucleus independent magnetic shieldings (NIMS) in the position of the atoms of the solute caused by the surrounding molecules. ${ }^{33}$

A similar approach can be employed for estimating contributions to NMR chemical shifts from more distant solvent molecules. When the position of the solute molecule relative to each solvent molecule is known, a single QM calculation can be performed on the solvent molecule as illustrated in Figure 1. By performing NIMS calculations in the relative position of the investigated solute atoms with respect to the solvent molecule, the shielding contribution can be obtained as a sum of the contributions from all solvent molecules. In addition, the contribution from all solvent configurations can be obtained in a single calculation on the solvent molecule. In this approach, it is assumed that the molecular structures of all solvent molecules are identical. They are of course not exactly identical. However, the long-ranged contribution is rather small implying that tiny errors in the molecular structure does not significantly affect the long-ranged current contribution to the NMR chemical shifts.

In this work, we investigate how the current contributions from adjacent and more distant molecules affect the magnetic shieldings and how the current densities of stacked aromatic and antiaromatic molecules changes due to the presence of the surrounding molecules. The aim of the study is to understand how to consider current contributions from aromatic 


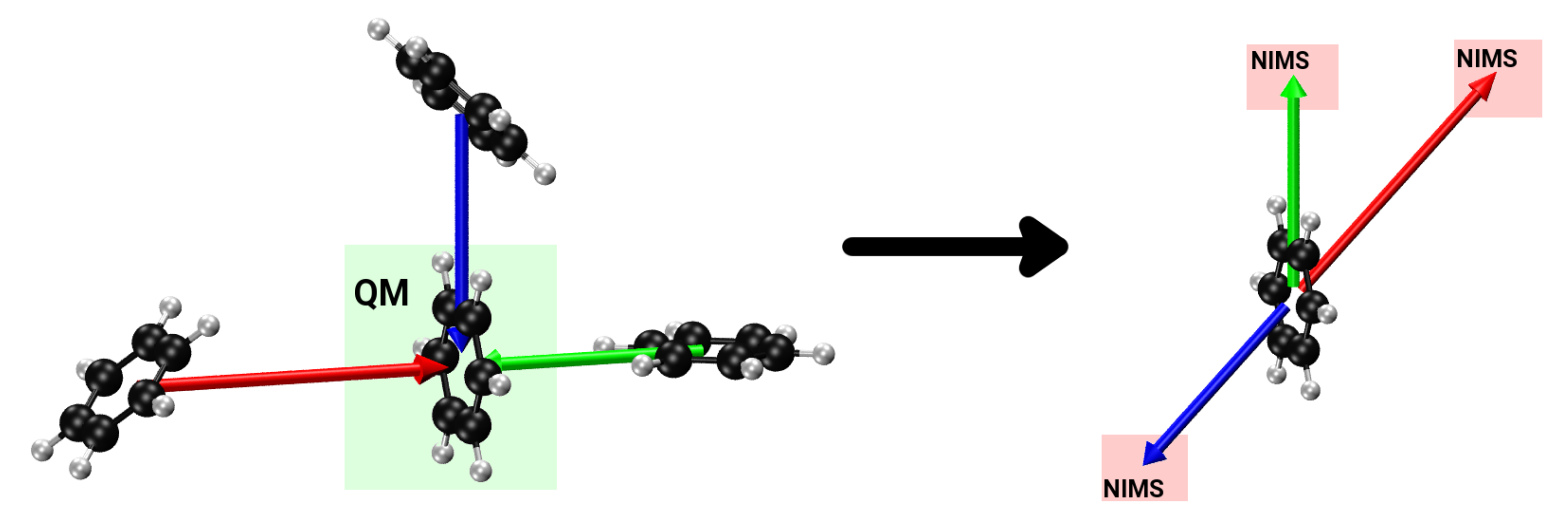

Figure 1: The method to calculate the magnetic shielding contributions from solvent molecules to a selected nucleus of the probe molecule is illustrated. The nucleus independent magnetic shieldings (NIMS) are calculated for one solvent molecule at the relative coordinates corresponding to a given nucleus of the probe molecule. All significant solvent molecules can be considered in one magnetic shielding calculation. The picture has been made using VMD 1.9.2 and Gimp 2.8. ${ }^{35}$

moieties in NMR chemical shift calculations on substances dissolved in a solvent or on molecules in the liquid and solid phases.

The computational levels are described in Section 2. The theory is briefly discussed in Section 3. The results of the shielding calculations are presented in Section 4 and the electrostatic effects are discussed in Section 5. Results of the current density calculations are reported in Section 6. The main conclusions are drawn in Section 7.

\section{Computational details}

The molecular structures of benzene, hexadehydro[12] annulene, dodecadehydro[18] annulene, and hexabenzocoronene were optimized at the density functional theory (DFT) level using Turbomole. ${ }^{36,37}$ The Perdew-Burke-Ernzerhof hybrid functional (PBE0) was employed in combination with the Karlsruhe split-valence (def2-SVP) basis set augmented with polarization functions. ${ }^{38-40}$ The optimized monomer structures were adopted when constructing the molecular stacks of benzene, hexadehydro[12]annulene, and dodecadehydro[18]annulene. 
The separation between the individual benzene molecules was chosen to $3.9 \AA$, which is close to the van der Waals minimum of the benzene dimer. ${ }^{41}$ The same procedure was used for constructing the hexadehydro[12]annulene and dodecadehydro[18]annulene stacks. For the hexabenzocoronene stacks, the separation distance between the individual molecules was adopted from the PBE0/def2-SVP/D3 optimization of the structure for the hexabenzocoronene trimer, where D3 denotes Grimme's semiempirical dispersion correction. ${ }^{42}$ The atomic positions of the two and four outer hexabenzocoronene molecules of the pentamer and the heptamer, respectively, were obtained by adopting the same intermolecular distance as for the trimer. The individual molecules as well as the molecular stacks of benzene, hexadehydro[12]annulene, and dodecadehydro[18]annulene, and hexabenzocoronene were assumed to belong to the $D_{6 \mathrm{~h}}, D_{3 \mathrm{~h}}, D_{3 \mathrm{~h}}$, and $D_{6}$ point groups, respectively. The enumeration of the molecules in the stacks begins symmetrically from the ends of the stack as shown in Figure 2 for the decamer. The molecule(s) in the middle has the highest number.

The nuclear magnetic shielding tensors were calculated with TURBomole at the DFT level using the Becke-Lee-Yang-Parr three-parameter hybrid functional (B3LYP), the m5 integration grid, and triple- $\zeta$ (def2-TZVP) basis sets. ${ }^{39,43-46}$

Magnetically induced current densities and ring-current susceptibilities were calculated using the GIMIC program, ${ }^{47-50}$ which is an independent program that uses the magnetically perturbed and unperturbed one-body density matrices and basis-set information as input data. ${ }^{47,48}$ The density matrices are obtained in NMR shielding calculations with Turbomole. The calculated current densities are origin independent with a fast basis-set convergence, because gauge-including atomic orbitals (GIAO) are employed. ${ }^{51-54}$ Explicit values for current strengths (susceptibilities) are obtained by numerical integration of the current density passing through cut planes perpendicularly to selected bonds of the molecular system. ${ }^{47}$ The ring-current susceptibility can be used as a reliable measure of molecular aromaticity. ${ }^{49}$ The sign and magnitude of the obtained ring-current strengths indicate whether molecular rings are aromatic, antiaromatic, or nonaromatic thus having diatropic, paratropic, or vanishing 
net ring current, respectively. ${ }^{34}$

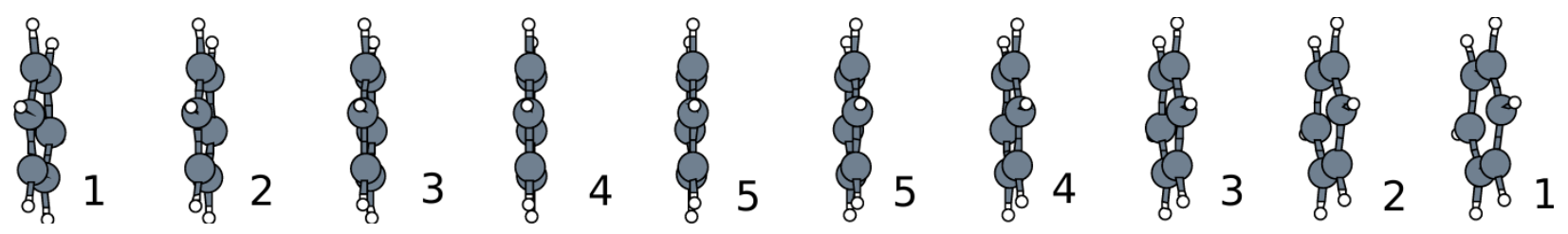

Figure 2: The enumeration of the stacked molecules of the decamer used in the graphs. The picture has been made using Xmakemol 5.16 and Gimp 2.8.

\section{Theory}

The magnetic shielding of the probe molecule $\left(\sigma_{\text {stack }}\right)$ can be divided into the following main contributions

$$
\sigma_{\text {stack }}=\sigma_{\text {monomer }}+\Delta \sigma_{\text {current }}+\sigma_{\text {current }}+\sigma_{\text {electrostatic }}+\sigma_{\text {relaxation }}
$$

where $\sigma_{\text {monomer }}$ is the shielding calculated for the monomer, $\Delta \sigma_{\text {current }}$ is due to changes in the current density of the studied molecule due the presence of the adjacent molecules. $\sigma_{\text {current }}$ and $\sigma_{\text {electrostatic }}$ are the current and electrostatic contributions from the adjacent molecules, respectively, and $\sigma_{\text {relaxation }}$ is the contribution due to structural changes of the molecule. $\sigma_{\text {relaxation }}$ vanishes here, because the structure of the individual molecules are fixed. Vibrational contributions have also been omitted. The $\sigma_{\text {electrostatic }}$ and $\Delta \sigma_{\text {current }}$ contributions are difficult to separate. However, they have different physical background. The $\Delta \sigma_{\text {current }}$ contribution originates from the confinement of the monomer wavefunctions between the two molecules, whereas $\sigma_{\text {electrostatic }}$ is due to electrostatic interactions with the atomic charges of the surrounding molecules. $\sigma_{\text {electrostatic }}$ should be possible to model by using point charges, whereas calculation of $\Delta \sigma_{\text {current }}$ requires a full QM treatment.

Aromatic and antiaromatic molecules have been chosen to maximize the current contribution to the magnetic shieldings. The magnetically induced ring currents of the adjacent (anti)aromatic molecules lead to a significant shift in the shieldings, whereas the electrostatic 
contribution is expected to be small for the nonpolar system. The electrostatic contributions have been estimated for one of the dimer molecules and for the central molecule of the pentamer stack by using point charges to describe the surrounding molecules. The ringcurrent contribution from more distant molecules can be estimated by performing nucleus independent magnetic shielding (NIMS) calculations in the position of the studied nucleus $\left(R_{I}\right)$ relative to the adjacent molecules. See Figure 1 The total ring-current correction for

the investigated molecule $\left(\sigma_{\text {current }}^{0}\right)$ is estimated by adding the individual NIMS contributions from all molecules outside the QM region.

$$
\sigma_{\text {current }}^{0}=\sum_{I \notin \mathrm{QM}} \sigma_{\mathrm{NIMS}}^{I}\left(R_{I}\right)
$$

The long-ranged solvent effects can be considered analogously, by averaging over a large number of solvent configurations taken from molecular dynamics simulations. However, here we have considered only one molecular structure for each stack.

\section{Shielding Calculations}

The current-density and ring-current contributions to the nuclear magnetic shieldings of benzene, hexadehydro[12]annulene, dodecadehydro[18]annulene, and hexabenzocoronene stacks have been analyzed by performing four series of calculations

1. The nuclear magnetic shieldings are calculated for the individual molecules in the molecular stacks. The obtained shieldings are compared to the corresponding shieldings calculated for a single molecule (monomer).

2. The deviations between the monomer shieldings and the shieldings for the molecules in the stacks are corrected for the ring-current contributions of the surrounding molecules by adding NIMS corrections.

3. The nuclear magnetic shieldings are calculated for the individual molecules in the 
molecular stacks. The obtained shieldings are compared to the corresponding shieldings calculated for a trimer. The shieldings of the first molecule in the stack are compared to the shieldings of the outer molecules of the trimer, whereas the shieldings of the rest of the molecules are compared to the shielding of the middle molecule of the trimer.

4. The deviations between the trimer shieldings and the shieldings for the molecules in the stack are corrected for the ring-current contributions from the molecules surrounding the trimer probe. The shieldings of the trimer are calculated at the QM level, whereas the ring-current contributions to the shieldings are estimated using NIMS.

Benzene and dodecadehydro[18]annulene represent aromatic molecules ${ }^{55}$ whereas hexadehydro[12]annulene is antiaromatic. ${ }^{55-57}$ Hexabenzocoronene consists of annelated conjugated rings that sustain diatropic or paratropic ring currents. ${ }^{15,18,47}$

\subsection{Benzene stacks}

The nucleus independent magnetic shielding (NIMS) calculations show that the ring-current contributions from the adjacent rings is about $1.7 \mathrm{ppm}$ for the ${ }^{13} \mathrm{C}$ NMR shieldings in the middle of the decamer stack of benzene molecules. However, the interaction between the nearest neighbors significantly affects the current density leading to a $85 \%$ cancellation of the long-ranged ring-current shielding effect. The 'Monomer' curve in Figure 3a shows the differences in the ${ }^{13} \mathrm{C}$ NMR shieldings as obtained for a benzene stack consisting of ten molecules. The numbers along the abscissa in Figure 3 denotes the position of the molecule in the stack. Thus, number 1 corresponds to the first molecule in the stack implying that the central molecule of the decamer is labelled 5. See the decamer numbering in Figure 2.

When the long-ranged ring-current contributions are added to the monomer shieldings, the NIMS corrected ${ }^{13} \mathrm{C}$ NMR shieldings systematically deviate by 2 ppm from the shieldings calculated at the full QM level for the stack. The 'Monomer+NIMS' curve in Figure 3a shows the corrected shielding differences obtained as described in case 2 above. The Trimer and 
Trimer+NIMS curves in Figure 3a shows the corresponding data with the trimer as starting point as described in cases 3 and 4 above, respectively. The shielding difference between the trimer calculation and those obtained for the decamer is of the same size but with opposite sign as compared to those obtained in the monomer calculation. When the NIMS correction is added to the trimer values, the largest deviation between the corrected ${ }^{13} \mathrm{C} N \mathrm{NR}$ shieldings and the ones obtained in the full QM calculation is about $-0.05 \mathrm{ppm}$. Negative difference values mean that the simulated shieldings are smaller than the fully calculated ones.

Similar calculations of the ${ }^{1} \mathrm{H}$ NMR shieldings show that the neighbor ring-current shielding effect for the benzene decamer is about $-1.2 \mathrm{ppm}$. When the NIMS correction is added to the ${ }^{1} \mathrm{H}$ NMR shielding of the monomer, the discrepancy to the fully QM value of the benzene decamer is about $-0.2 \mathrm{ppm}$. The largest deviation of the trimer calculation is -0.35 ppm, which becomes -0.07 ppm when adding the NIMS corrections. The four cases for the ${ }^{1} \mathrm{H}$ NMR shielding differences are shown in Figure 3b.

Comparing the shieldings of the inner benzene molecules with the outermost benzene molecule of the decamer shows that the shielding difference for the molecules with only one nearest neighbor differ significantly from shielding differences obtained for the molecules surrounded by two benzenes. To accurately simulate the shieldings of the benzene stacks, the nearest neighbor molecules have to be considered at the QM level, whereas the ringcurrent contributions from the rest of the molecules can to first approximation be considered by adding NIMS corrections from the other benzene molecules in the stack. Detailed graphs analogous to the ones in Figure 3 are reported for shorter benzene stacks in the Supporting Information.

\section{$4.2 \quad$ Hexadehydro[12] annulene stacks}

Hexadehydro[12]annulene is an antiaromatic molecule with $12 \pi$ electrons along the conjugation pathway around the 12-membered carbon ring. For antiaromatic molecules the long-ranged shielding contribution is negative (deshielding) in the cone above and below the 


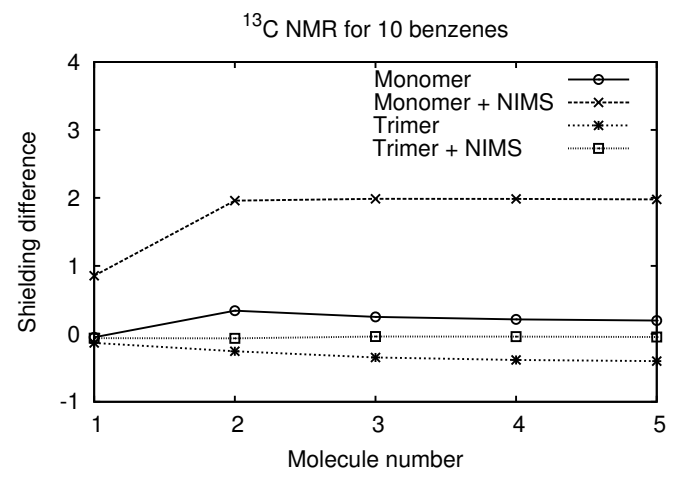

(a)

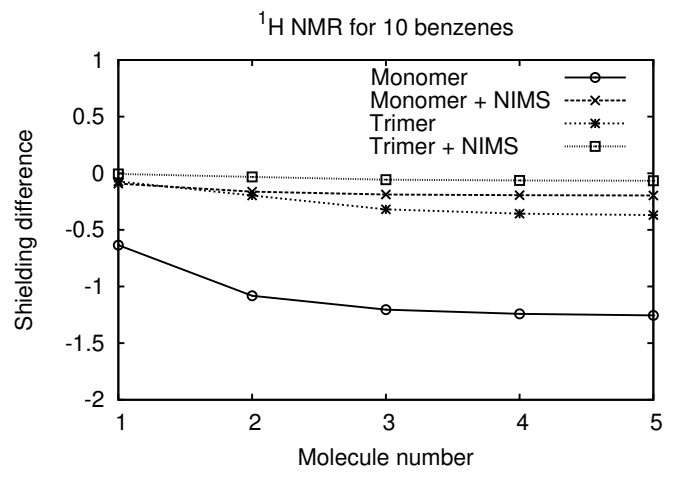

(b)

Figure 3: (a) ${ }^{13} \mathrm{C}$ NMR and (b) ${ }^{1} \mathrm{H}$ NMR shieldings of ten stacked benzene molecules are given for the individual molecules in the decamer. The number on the abscissa enumerates the molecules beginning from the end of the stack. The graph shows the shielding difference between the decamer and the monomer (Monomer) as described in case (1), and (Monomer+NIMS) as in case (2), where the rest of the molecules is considered by NIMS corrections. The graph also shows the shielding difference between the decamer and the trimer (Trimer) and as in case (3) and with the rest of the molecules is considered by NIMS corrections (Trimer+NIMS) as described in the text as case (4). The graphs have been made using Gnuplot 4.6.

molecular plane. ${ }^{22}$ The differences between the ${ }^{13} \mathrm{C}$ NMR and ${ }^{1} \mathrm{H}$ NMR shieldings at the $\mathrm{C}=\mathrm{C}$ double bond of the hexadehydro[12] annulene stacks and the corresponding shieldings for the monomer are shown in Figure 4a and Figure 4b, respectively. The carbon shieldings of the monomer differ by 6-7 ppm from those calculated for the decamer and the difference in the ${ }^{1} \mathrm{H}$ NMR shieldings is about $2 \mathrm{ppm}$. By adding the NIMS corrections (case 2), the largest deviation for the hydrogen shielding is $-0.2 \mathrm{ppm}$ as shown by the 'Monomer+NIMS' curve in Figures 4b. The NIMS corrections reduce the deviation in the ${ }^{13} \mathrm{C}$ NMR shielding to $1.6 \mathrm{ppm}$ as shown by the 'Monomer+NIMS' curve in Figure 4a.

The corresponding trimer calculations (case 3 and 4) are also shown with the Trimer and Trimer+NIMS curves in Figures $4 \mathrm{a}$ and $4 \mathrm{~b}$. Comparison of the shielding values of the trimer and the decamer in Figure 4 shows that the largest deviation obtained for the decamer is about 1 ppm. By adding the long-ranged ring-current contributions estimated from the NIMS calculations to the trimer shieldings one obtains agreements of $-0.1 \mathrm{ppm}$ for the hydrogen shieldings and the carbon shieldings agree within $0.4 \mathrm{ppm}$ for the decamer. Graphs reporting 
the shielding differences for all atoms of the studied hexadehydro[12]annulene stacks are reported as Supporting Information.

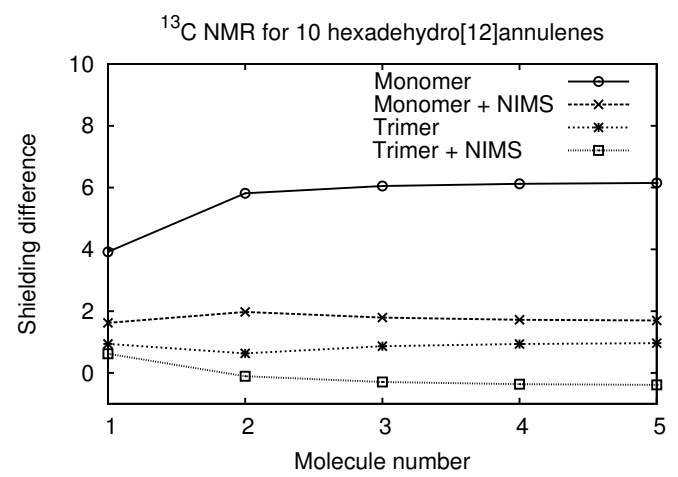

(a)

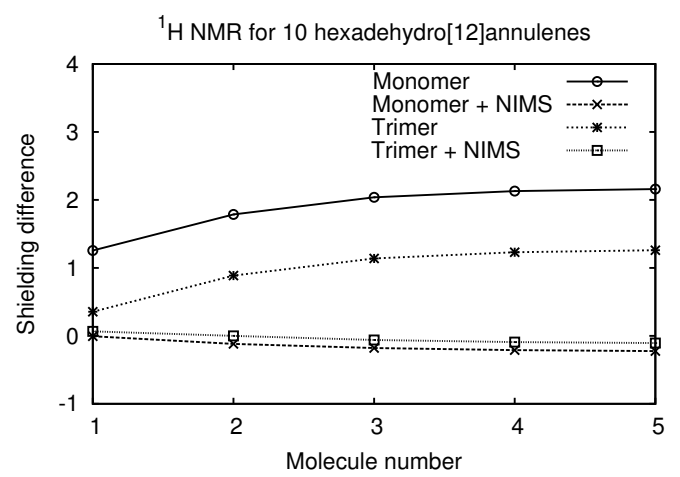

(b)

Figure 4: (a) ${ }^{13} \mathrm{C}$ NMR and (b) ${ }^{1} \mathrm{H}$ NMR shieldings at the $\mathrm{C}=\mathrm{C}$ double bond of the ten stacked hexadehydro[12] annulene molecules are given for the individual molecules in the decamer. The number on the abscissa enumerates the molecules beginning from the end of the stack. The graph shows the shielding difference between the decamer and the monomer (Monomer) as described in case (1), and (Monomer+NIMS) as in case (2), where the rest of the molecules is considered by NIMS corrections. The graph also shows the shielding difference between the decamer and the trimer (Trimer) and as in case (3) and with the rest of the molecules is considered by NIMS corrections (Trimer+NIMS) as described in the text as case (4). The graphs have been made using Gnuplot 4.6.

\subsection{Dodecadehydro[18]annulene stacks}

Dodecadehydro[18]annulene is an aromatic molecule with $18 \pi$ electrons along the conjugation pathway around the 18-membered carbon ring. The carbon shieldings for the $\mathrm{sp}^{2}$ hybridized carbon are shown in Figure 5a. The 'Monomer' curve in Figure 5a shows that the deviations for the ${ }^{13} \mathrm{C}$ NMR shieldings obtained in the monomer calculations are in the range of $-1 \mathrm{ppm}$ to $-3 \mathrm{ppm}$. The deviation increases with the position in the stack and is not converged even for the molecule in the middle of the decamer. The 'Monomer+NIMS' curve in Figure 5a shows that more systematic values are obtained when considering the NIMS contributions from the other rings. The shielding deviations of the monomer plus NIMS calculations are almost as large as the absolute shielding differences obtained when comparing 
with the uncorrected monomer shieldings. The largest deviations obtained in the trimer calculation is about $2 \mathrm{ppm}$. Considering the NIMS correction in combination with the trimer calculations leads to shielding values that agree within $1 \mathrm{ppm}$ with the carbon shieldings calculated for the whole dodecadehydro[18]annulene decamer. Detailed graphs reporting the shielding differences for the atoms of the all the studied dodecadehydro[18]annulene stacks are given in the Supporting Information.

The difference between the ${ }^{1} \mathrm{H}$ NMR shieldings of the monomer and the individual molecules of the dodecadehydro[18]annulene decamer increases systematically from -0.5 ppm for the molecule at the end of the stack to about $-3.0 \mathrm{ppm}$ in the middle of it. See Figure 5b. For the molecules at the end of the stack, the ring-current shieldings differ from those in the middle of the decamer, because the molecule at the end of the stack has only one adjacent molecule. For the dodecadehydro[18]annulene decamer, the deviating ring-current shielding for the molecules at the end of the stack penetrates far into the stack, because the large radius of the ring current leads to more a long-ranged ring-current shielding than for the smaller rings. For the hydrogens, the NIMS corrected monomer shieldings agree within $0.5 \mathrm{ppm}$ with the corresponding values calculated for the molecular stacks. The largest deviation is obtained in the middle of the decamer. The deviations obtained in the calculations on the trimer are about half the ones obtained for the monomer, whereas the shielding differences of the NIMS corrected trimer is about the same as obtained in the NIMS corrected monomer calculation shown with the 'Monomer+NIMS' and 'Trimer+NIMS' curves in Figure 5b, respectively. The NIMS correction is not as accurate for the hydrogen shieldings of the dodecadehydro[18]annulene stacks as for the smaller molecular rings. The agreement is not significantly better when the pentamer is taken as the starting point either. The long-ranged contributions have to be considered to obtain satisfactory agreement with the full QM calculation. The corresponding graph for the pentamer is shown in the Supporting Information. 


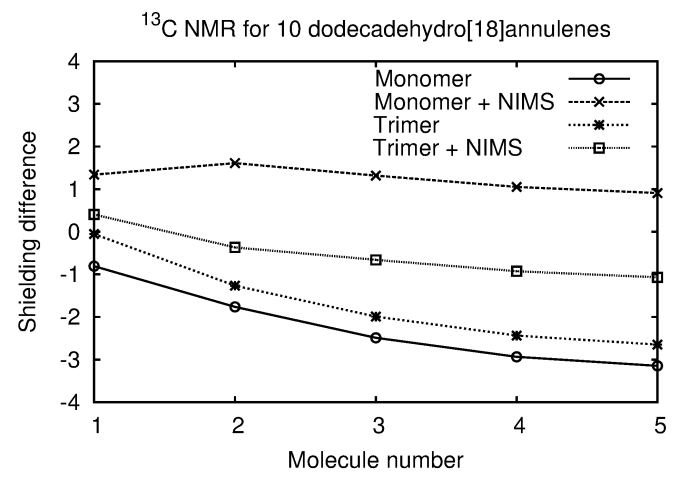

(a)

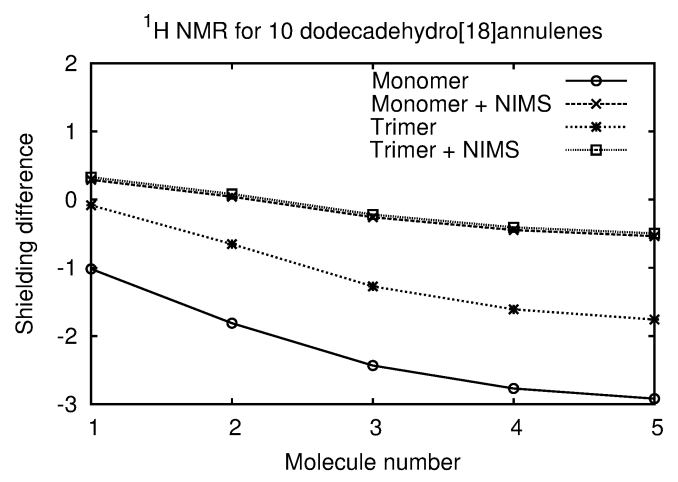

(b)

Figure 5: (a) ${ }^{13} \mathrm{C}$ NMR and (b) ${ }^{1} \mathrm{H}$ NMR shieldings at the $\mathrm{C}=\mathrm{C}$ double bond of the of ten stacked dodecadehydro[18]annulene molecules are given for the individual molecules in the decamer. The number on the abscissa enumerates the molecules beginning from the end of the stack. The graph shows the shielding difference between the decamer and the monomer (Monomer) as described in case (1), and (Monomer+NIMS) as in case (2), where the rest of the molecules is considered by NIMS corrections. The graph also shows the shielding difference between the decamer and the trimer (Trimer) and as in case (3) and with the rest of the molecules is considered by NIMS corrections (Trimer+NIMS) as described in the text as case (4). The graphs have been made using Gnuplot 4.6.

\subsection{Hexabenzocoronene stacks}

The ${ }^{1} \mathrm{H}$ NMR and ${ }^{13} \mathrm{C}$ NMR shieldings of the hexabenzocoronene monomer and trimer were calculated at the B3LYP level using the def2-TZVP basis sets. The shieldings for the trimer were also estimated by adding NIMS contributions calculated for the monomer to the monomer shieldings. The differences between the monomer shieldings and the shieldings of the inner and outer molecules of the trimer as well as the the NIMS simulated shieldings for the trimer are shown in Figure 6a. The figure shows that the adjacent molecules cause large changes in the carbon shieldings, which cannot be simulated by calculating nucleus independent magnetic shieldings (NIMS) for the monomer. The shieldings obtained in the NIMS calculations deviate rather systematically up to $5 \mathrm{ppm}$ from the values obtained in the trimer calculation. The largest deviations are obtained for the carbons at the center of the hexabenzocoronene, whereas for the hydrogen shieldings the NIMS calculations yield a rough estimate for the chemical shifts due to the adjacent molecules. The calculations have 
to be performed using large basis set, since calculations at the B3LYP level using def2-SVP basis set yielded qualitatively different results as seen when comparing the graphs in Figure $6 \mathrm{a}$ with those in Figure 6b.

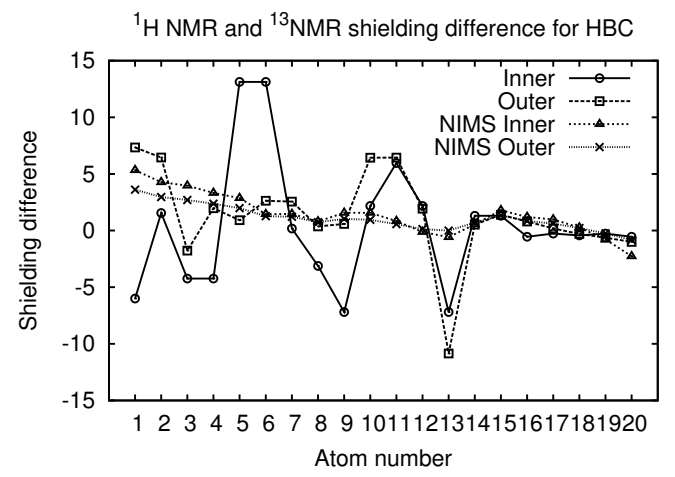

(a)

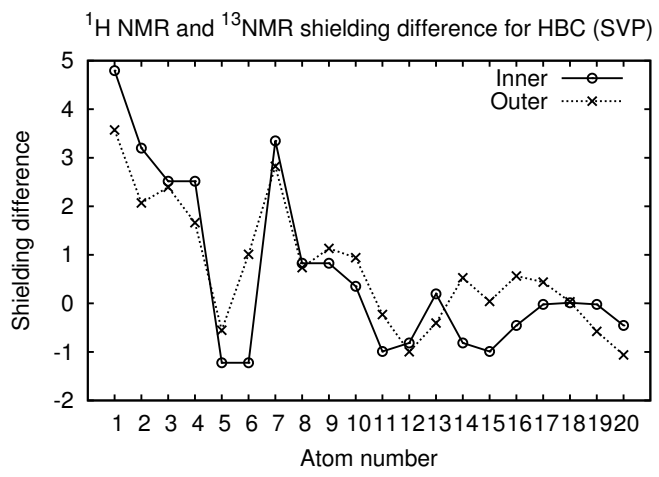

(b)

Figure 6: (a) The differences between ${ }^{1} \mathrm{H}$ NMR and ${ }^{13} \mathrm{C}$ NMR shieldings calculated at the B3LYP/def2-TZVP level for a hexabenzocoronene (HBC) molecule and the corresponding shieldings calculated for the inner and outer molecules of the hexabenzocoronene trimer. The difference between calculated trimer shift and the NIMS simulated shielding contributions are also shown for the inner and outer molecules of the trimer. (b) The differences between ${ }^{1} \mathrm{H}$ NMR and ${ }^{13} \mathrm{C}$ NMR shieldings calculated at the B3LYP/def2-SVP level for a hexabenzocoronene molecule and the corresponding shieldings calculated for the inner and outer molecules of the hexabenzocoronene trimer. Lower atom numbers corresponds to the symmetry unique carbons in the inner part of the molecule and the 7 last atoms are the symmetry unique hydrogens at the outer edge. The graphs have been made using Gnuplot 4.6.

\section{$5 \quad$ Electrostatic interactions}

The electrostatic interaction of the adjacent molecules is taken into account by using point charges for the carbons and the hydrogens. The point charges were determined using Kollman's electrostatic point approach. ${ }^{58}$ The following point charges were employed: $\mathrm{q}_{\mathrm{C}}$ $=-0.1244$ and $\mathrm{q}_{\mathrm{H}}=0.1244$ for benzene; $\mathrm{q}_{\mathrm{C}(\text { double })}=-0.1060, \mathrm{q}_{\mathrm{C}(\text { triple })}=-0.0260$, and $\mathrm{q}_{\mathrm{H}}=$ 0.1320 for hexadehydro[12] annulene; $\mathrm{q}_{\mathrm{C}(\text { double })}=-0.1045, \mathrm{q}_{\mathrm{C}(\text { triple })}=-0.0015, \mathrm{q}_{\mathrm{C}(\text { triple })}=-0.0303$ (central), and $\mathrm{q}_{\mathrm{H}}=0.1363$ for dodecadehydro[18]annulene.

The calculation with point charges were performed on the dimer, where one molecule was 
represented by point charges. The pentamers were also studied by using point charges. The two outermost molecules of the pentamers were represented by point charges. The magnetic shieldings were calculated for the molecule in the middle of the trimer that was treated quantum mechanically.

For benzene, the electrostatic shifts are very small. For the carbons, the shifts are even in the wrong direction leading to a larger deviation from the shielding obtained in the dimer calculation than when the electrostatic contributions are omitted. For the hydrogens the electrostatic shifts improve the agreement with the shieldings obtained in the dimer and pentamer calculations.

For the hexadehydro[12] annulenes, the electrostatic contribution seems to have the wrong sign for the shieldings of the $\mathrm{C}=\mathrm{C}$ carbons and the hydrogens. However, for the dimer the electrostatic shift improves the agreement with the dimer shielding when also the ringcurrent contribution is taken into account. For the dimer, the $\mathrm{C} \equiv \mathrm{C}$ carbons the electrostatic correction has the correct sign but is too small leading to a rather large deviation from the dimer shielding. For the pentamer, the electrostatic shift of the $\mathrm{C} \equiv \mathrm{C}$ carbons is slightly too large leading to a larger deviation than when only the ring-current contributions are considered.

For the dimer of the dodecadehydro[18]annulene, the electrostatic correction to the hydrogen shielding is about a factor of two too large, whereas for the $\mathrm{C} \equiv \mathrm{C}$ carbons, the electrostatic corrections are a factor of 2-3 too small. For the $\mathrm{C}=\mathrm{C}$ carbons, the electrostatic shift increases the deviation with the shieldings calculated for the dimer. For the pentamer, the electrostatic contributions to the $\mathrm{H}$ shieldings, the shieldings of the outer $\mathrm{C} \equiv \mathrm{C}$ carbons, and the shieldings of the $\mathrm{C}=\mathrm{C}$ carbons are too small, whereas for the central $\mathrm{C} \equiv \mathrm{C}$ carbons the electrostatic contribution is too large even though it is very small. In that case, a better agreement with the shieldings obtained in the calculations on the pentamer is obtained when omitting the electrostatic contributions. The different contributions to the magnetic shieldings of the dimer and pentamer are shown in Figure 7. 


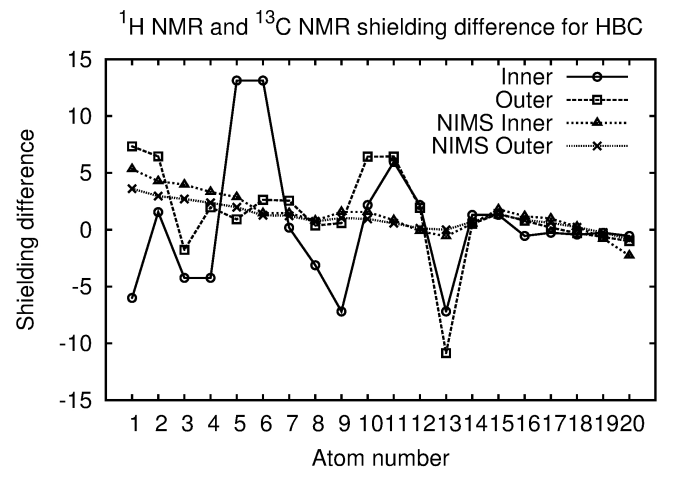

(a)

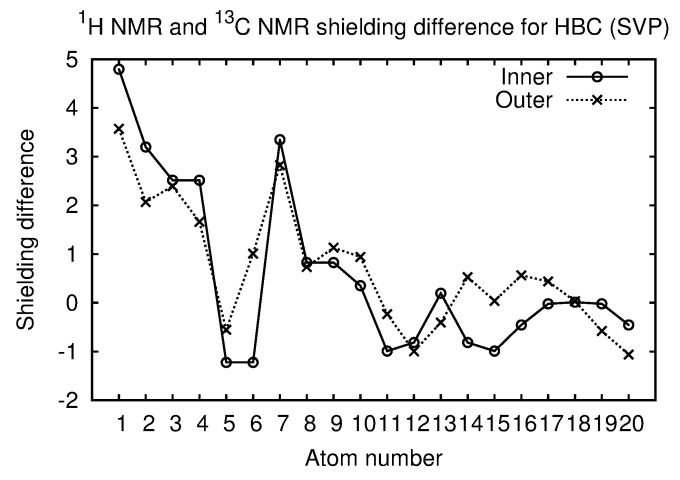

(b)

Figure 7: The deviation from the exact shieldings for (a) the dimers and (b) the pentamers. Case (A) shows the deviation between the shieldings obtained for the molecular stack and the shieldings of the monomer. (B) as in case (A), but with the monomer shieldings corrected for electrostatic interactions introduced by point charges. In case $(\mathrm{C})$, the current contribution is estimated by using the nucleus independent shielding correction from the neighbor has been added to the values of case (A). In case (D), both electrostatic contributions obtained using point charges and the current correction are added to the values obtained for case (A). In case (E), the shieldings obtained in the calculation on the molecular stack. For the pentamer the trimer is the starting point. The pentamer data are given for the shieldings of the central molecule. The graphs have been made using Gnuplot 4.6.

\section{Current density calculations}

After the ring-current effects originating from the neighbor molecules have been considered by NIMS corrections, the main reason for the remaining deviation in the magnetic shieldings is the detailed changes in the current density due to the presence of the neighbor molecules. The vector potential of the nuclear magnetic moments is proportional to $(\mathbf{m} \times \mathbf{r}) /|\mathbf{r}|^{3}$, where $\mathbf{m}$ denotes the three components of the magnetic moment and $\mathbf{r}$ is the distance from the nucleus. This implies that small changes in the current density in the vicinity of the studied molecule significantly affect the shieldings. Thus, the current contributions from nearest neighbors have to be estimated by performing calculations on two or three molecules in the stack.

Current density calculations on the benzene dimer show that the net strength of the ring current is not much affected by the presence of the adjacent molecules. Electrostatic effects are not important, because simulating the other molecule of the benzene dimer using 
point charges leads to almost no change in the magnetic shieldings. The main reason for the dimer shift in the magnetic shieldings is the confinement of the ring current between the rings as shown in Figure 8. The shape of the current density slightly differs from the linear combination of the two monomers. The plot in Figure 8 shows that the current density is somewhat weaker between the two benzene molecules than the sum of the current density for two benzenes. Since the changes in the current density occur relatively near the studied molecule, small changes in the current density may have a significant effect on the shieldings. The relation between the elements of the magnetic shielding tensor of nucleus $I\left(\sigma_{\alpha \delta}^{I}\right)$ and the current density tensor $\left(J_{\gamma}^{B_{\delta}}(\mathbf{r})\right)$ is given by the Biot-Savart expression ${ }^{59}$

$$
\sigma_{\alpha \delta}^{I}=-\frac{1}{c} \epsilon_{\alpha \beta \gamma} \int J_{\gamma}^{B_{\delta}}(\mathbf{r}) \frac{\left(r_{\beta}-R_{I \beta}\right)}{\left|\mathbf{r}-\mathbf{R}_{I}\right|^{3}} d \mathbf{r}
$$

where $c$ is the speed of light, $\alpha, \beta, \gamma, \delta$ denote Cartesian directions, $\epsilon_{\alpha \beta \gamma}$ is the Levi-Civita symbol, $B_{\delta}$ is the external magnetic field, and $\frac{\left(r_{\beta}-R_{I \beta}\right)}{\left|\mathbf{r}-\mathbf{R}_{I}\right|^{3}}$ is the vector potential for the magnetic interaction of the nuclear magnetic moment of nucleus $I$.

The ring-current susceptibility of the benzene dimer is $23.92 \mathrm{nA} / \mathrm{T}$ yielding a ring current strength of $11.96 \mathrm{nA} / \mathrm{T}$ in each molecule. Calculations on the benzene decamer yielded a total ring current of $118.70 \mathrm{nA} / \mathrm{T}$ or $11.87 \mathrm{nA} / \mathrm{T} /$ benzene molecule. The superposition of two benzene molecules yields a current strength of $24.04 \mathrm{nA} / \mathrm{T}$. Thus, the interaction between the molecules leads to a slightly weaker current strength of $0.12 \mathrm{nA} / \mathrm{T}$. However, more important is the redistribution of the current density shown in Figure 8a. For hexadehydro[12]annulene and dodecadehydro[18]annulene, the ring current strengths of the individual molecules are also rather independent of the length of the stack. For hexadehydro[12]annulene, the ring current strength of the individual molecules in the monomer, dimer and the decamer is $-25.43 \mathrm{nA} / \mathrm{T},-25.58 \mathrm{nA} / \mathrm{T}$, and $-25.64 \mathrm{nA} / \mathrm{T}$, respectively. For dodecadehydro[18]annulene, the corresponding current strengths are $17.40 \mathrm{nA} / \mathrm{T}, 17.50 \mathrm{nA} / \mathrm{T}$ and $17.81 \mathrm{nA} / \mathrm{T}$. The current density difference in Figure 8b shows that the paratropic ring current in the hexadehydro[12]annulene dimer is slightly stronger than for the monomer, 
whereas for dodecadehydro[18]annulene the current density difference shown in Figure 8c is less systematic. The shorter distance between the molecules of the hexabenzocoronene dimer leads to larger changes in the current density between the two molecules as seen in Figure 8d. This is the basic reason why the NIMS correction fails for hexabenzocoronene.

\section{Conclusions}

The calculations of the ${ }^{1} \mathrm{H}$ NMR and ${ }^{13} \mathrm{C}$ NMR shieldings for the benzene stacks suggest that the ring-current contributions from more distant molecules can be estimated using NIMS calculations, whereas the nearest neighbors must be included in the shielding calculations, because small changes in the current density in the vicinity of the probe molecule significantly affect the shieldings. The neighbor molecules affect the shape of the current density even though the current strengths of the individual molecules are little affected. The calculations on the trimers with the shielding contribution of the rest of the molecules considered using NIMS leads to rather accurate estimates for the shieldings of the molecules in the stacks. Electrostatic effects are small for the nonpolar molecules. Because the size of the electrostatic corrections is small the corrected shieldings are in some cases in better agreement with the shieldings obtained in the fully quantum mechanical calculations. Thus, the uncertainty of the electrostatic corrections are for these nonpolar molecules larger than the electrostatic shifts.

\section{Acknowledgement}

This work has been supported by projects 266227 and 275845 of the Academy of Finland. We acknowledge Magnus Ehrnrooth Foundation for financial support. DS thanks the Swedish Cultural Foundation in Finland, the Alexander von Humboldt Stiftung, and the Fulbright Foundation for financial support during his sabbatical leave. CO thanks the DFG project Oc35/4-1 for financial support. The CONICYT-AKA-ERNC-001 project is acknowledged for 
financial support. CSC - the Finnish IT Center for Science - is acknowledged for computer time.

\section{Supporting Information Available}

Electronic supplementary information (ESI) available: Cartesian coordinates and nuclear magnetic shieldings of the studied molecules. This material is available free of charge via the Internet at http://pubs.acs.org/. 


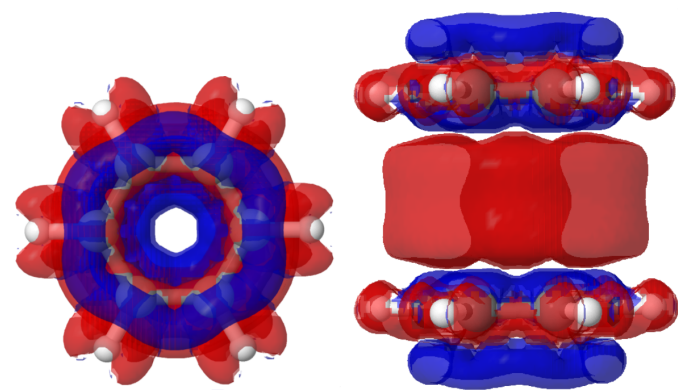

(a)

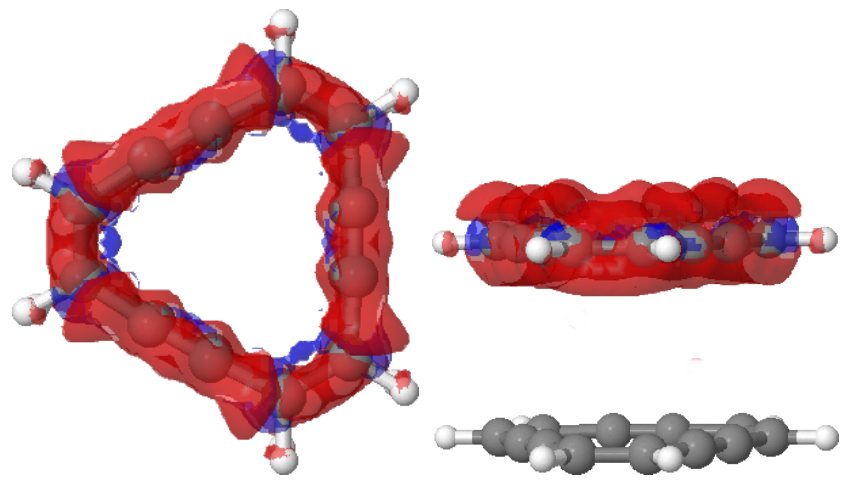

(b)

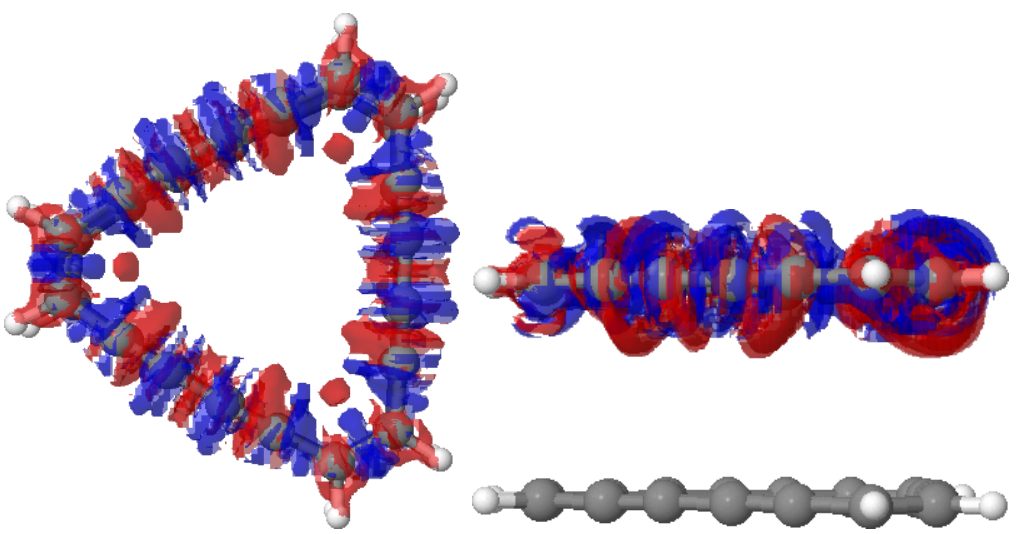

(c)
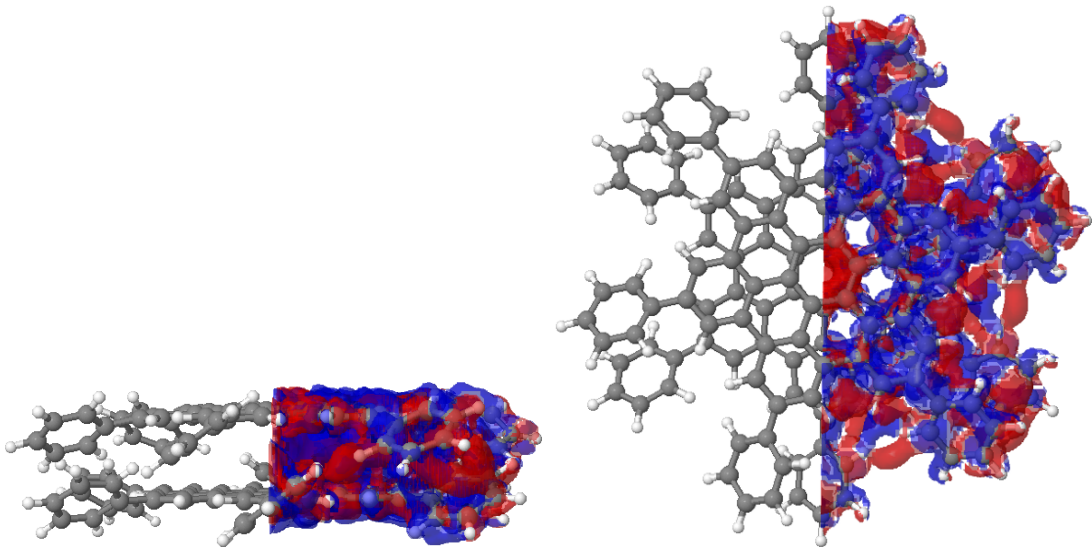

(d)

Figure 8: The current density difference between the dimer and the superposition of two monomers. Positive current density difference is blue and the negative one is red. The current density difference is shown for (a) benzene (b) hexadehydro[12] annulene (c) dodecadehydro[18] annulene and (d) hexabenzocoronene. Negative values corresponds to weaker current density in the dimer. The cut-off for benzene is a factor of five smaller than for the rest of the molecules, whose cut-off threshold is 0.0005. The pictures have been made using Jmol 12.2 and Gimp 2.8. 


\section{References}

(1) Senn, H. M.; Thiel, W. QM/MM Methods for Biomolecular Systems. Angew. Chem. Int. Ed. 2009, 48, 1198-1229.

(2) Standara, S.; Kulhnek, P.; Marek, R.; Straka, M. ${ }^{129}$ Xe NMR chemical shift in Xe@C60 calculated at experimental conditions: Essential role of the relativity, dynamics, and explicit solvent. J. Comp. Chem. 2013, 34, 1890-1898.

(3) Özcan, N.; Mares, J.; Sundholm, D.; Vaara, J. Solvation chemical shifts of perylenic antenna molecules from molecular dynamics simulations. Phys. Chem. Chem. Phys. 2014, 16, 22309-22320.

(4) Flaig, D.; Beer, M.; Ochsenfeld, C. Convergence of Electronic Structure with the Size of the QM Region: Example of QM/MM NMR Shieldings. J. Chem. Theory Comput. 2012, 8, 2260-2271.

(5) Wesołowski, T. A.; Warshel, A. Frozen density functional approach for ab initio calculations of solvated molecules. J. Phys. Chem. 1993, 97, 8050-8053.

(6) Neugebauer, J.; Louwerse, M. J.; Baerends, E. J.; Wesołowski, T. A. The merits of the frozen-density embedding scheme to model solvatochromic shifts. J. Chem. Phys. 2005, 122, 094115.

(7) Shedge, S. V.; Wesołowski, T. A. Nonuniform Continuum Model for Solvatochromism Based on Frozen-Density Embedding Theory. ChemPhysChem 2014, 15, 3291-3300.

(8) Klamt, A.; Schüürmann, G. COSMO: a new approach to dielectric screening in solvents with explicit expressions for the screening energy and its gradient. J. Chem. Soc., Perkin Trans. 2 1993, 799-805.

(9) Schäfer, A.; Klamt, A.; Sattel, D.; Lohrenz, J. C. W.; Eckert, F. COSMO Implementa- 
tion in TURBOMOLE: Extension of an efficient quantum chemical code towards liquid systems. Phys. Chem. Chem. Phys. 2000, 2, 2187-2193.

(10) Miertus, S.; Scrocco, E.; Tomasi, J. Electrostatic interaction of a solute with a continuum. A direct utilization of ab initio molecular potentials for the prevision of solvent effects. Chem. Phys. 1981, 55, 117-129.

(11) Tomasi, J.; Mennucci, B.; Cammi, R. Quantum Mechanical Continuum Solvation Models. Chem. Rev. 2005, 105, 2999-3094.

(12) Taubert, S.; Konschin, H.; Sundholm, D. Computational studies of ${ }^{13} \mathrm{C}$ NMR chemical shifts of saccharides. Phys. Chem. Chem. Phys. 2005, 7, 2561-2569.

(13) Zhu, T.; Zhang, Z. H., John; He, X. Automated Fragmentation QM/MM Calculation of Amide Proton Chemical Shifts in Proteins with Explicit Solvent Model. J. Chem. Theory Comput. 2013, 9, 2104-2114.

(14) Ochsenfeld, C. An ab initio study of the relation between NMR chemical shifts and solid-state structures: hexabenzocoronene derivatives. Phys. Chem. Chem. Phys. 2000, 2, 2153-2159.

(15) Ochsenfeld, C.; Brown, S. P.; Schnell, I.; Gauss, J.; Spiess, H. W. Structure Assignment in the Solid State by the Coupling of Quantum Chemical Calculations with NMR Experiments: A Columnar Hexabenzocoronene Derivative. J. Am. Chem. Soc. 2001, 123, 2597-2606.

(16) Brown, S. P.; Schaller, T.; Seelbach, U. P.; Koziol, F.; Ochsenfeld, C.; Klärner, F.G.; Spiess, H. W. Structure and Dynamics of the Host-Guest Complex of a Molecular Tweezer: Coupling Synthesis, Solid-State NMR, and Quantum-Chemical Calculations. Angew. Chem. Int. Ed. 2001, 40, 717-720. 
(17) Ochsenfeld, C.; Koziol, F.; Brown, S. P.; Schaller, T.; Seelbach, U. P.; Klärner, F.-G. A Study of a Molecular Tweezer Host-Guest System by a Combination of QuantumChemical Calculations and Solid-State NMR Experiments. Solid State Nucl. Mag. 2002, 22, 128-153.

(18) Pisula, W.; Tomovic, Z.; Watson, M. D.; Müllen, K.; Kussmann, J.; Ochsenfeld, C.; Metzroth, T.; Gauss, J. Helical Packing of Discotic Hexa-Phenyl Hexa-perihexabenzocoronenes: Theory and Experiment. J. Phys. Chem. B 2007, 111, 74817487.

(19) Zienau, J.; Kussmann, J.; Koziol, F.; Ochsenfeld, C. Molecular recognition in molecular tweezers systems: quantum-chemical calculation of NMR chemical shifts. Phys. Chem. Chem. Phys. 2007, 9, 4552-4562.

(20) Schaller, T.; Büchele, U. P.; Klärner, F.-G.; Bläser, D.; Boese, R.; Brown, S. P.; Spiess, H. W.; Koziol, F.; Kussmann, J.; Ochsenfeld, C. Structure of Molecular Tweezer Complexes in the Solid State: NMR Experiments, X-ray Investigations, and Quantum Chemical Calculations. J. Am. Chem. Soc. 2007, 129, 1293-1303.

(21) Zienau, J.; Kussmann, J.; Ochsenfeld, C. Quantum-chemical simulation of solid-state NMR spectra: the example of a molecular tweezer host-guest complex. Mol. Phys. 2010, 108, 333-342.

(22) Wüthrich, K. NMR Studies of Structure and Function of Biological Macromolecules (Nobel Lecture). Angew. Chem. Int. Ed. 2003, 42, 3340-3363.

(23) Flaig, D.; Beer, M.; Ochsenfeld, C. Convergence of Electronic Structure with the Size of the QM Region: Example of QM/MM NMR Shieldings. J. Chem. Theory Comput. 2012, 8, 2260-2271.

(24) Sumowski, C. V.; Hanni, M.; Schweizer, S.; Ochsenfeld, C. Sensitivity of Ab Initio vs 
Empirical Methods in Computing Structural Effects on NMR Chemical Shifts for the Example of Peptides. J. Chem. Theory Comput. 2014, 10, 122-133.

(25) Aidas, K.; Møgelhøj, A.; Kjær, H.; Nielsen, C. B.; Mikkelsen, K. V.; Ruud, K.; Christiansen, O.; Kongsted, J. Solvent Effects on NMR Isotropic Shielding Constants. A Comparison between Explicit Polarizable Discrete and Continuum Approaches. J. Phys. Chem. A 2007, 111, 4199-4210.

(26) He, X.; Wang, B.; Merz, K. M. Protein NMR Chemical Shift Calculations Based on the Automated Fragmentation QM/MM Approach. J. Phys. Chem. B 2009, 113, 1038010388.

(27) Bjornsson, R.; Bühl, M. Modeling Molecular Crystals by QM/MM: Self-Consistent Electrostatic Embedding for Geometry Optimizations and Molecular Property Calculations in the Solid. J. Chem. Theory Comput. 2012, 8, 498-508.

(28) Steinmann, C.; Olsen, J. M. H.; Kongsted, J. Nuclear Magnetic Shielding Constants from Quantum Mechanical/Molecular Mechanical Calculations Using Polarizable Embedding: Role of the Embedding Potential. J. Chem. Theory Comput. 2014, 10, 981988.

(29) Kussmann, J.; Ochsenfeld, C. Linear-scaling method for calculating nuclear magnetic resonance chemical shifts using gauge-including atomic orbitals within Hartree-Fock and density-functional theory. J. Chem. Phys. 2007, 127, 054103:1-16.

(30) Ochsenfeld, C.; Kussmann, J.; Koziol, F. Ab Initio NMR Spectra for Molecular Systems with a Thousand and More Atoms: A Linear-Scaling Method. Angew. Chem. Int. Ed. 2004, 43, 4485-4489.

(31) Maurer, M.; Ochsenfeld, C. A linear- and sublinear-scaling method for calculating NMR shieldings in atomic orbital-based second-order Møller-Plesset perturbation theory. J. Chem. Phys. 2013, 138, 174104:1-15. 
(32) Beer, M.; Kussmann, J.; Ochsenfeld, C. Nuclei-selected NMR shielding calculations: A sublinear-scaling quantum-chemical method. J. Chem. Phys. 2011, 134, 074102:1-15.

(33) Heine, T.; Corminboeuf, C.; Grossmann, G.; Haeberlen, U. Proton magnetic shielding tensors in benzene - From the individual molecule to the crystal. Angew. Chem. Int. Ed. 2006, 45, 7292-7295.

(34) Fliegl, H.; Sundholm, D.; Taubert, S.; Jusélius, J.; Klopper, W. Magnetically Induced Current Densities in Aromatic, Antiaromatic, Homoaromatic, and Nonaromatic Hydrocarbons. J. Phys. Chem. A 2009, 113, 8668-8676.

(35) Humphrey, W.; Dalke, A.; Schulten, K. VMD: Visual molecular dynamics. J. Mol. Graphics 1996, 14, 33-38.

(36) Ahlrichs, R.; Bär, M.; Häser, M.; Horn, H.; Kölmel, C. Electronic Structure Calculations on Workstation Computers: The Program System TURBOMOLE. Chem. Phys. Letters 1989, 162, 165-169.

(37) Furche, F.; Ahlrichs, R.; Hättig, C.; Klopper, W.; Sierka, M.; Weigend, F. Turbomole. WIREs Comput. Mol. Sci. 2014, 4, 91-100.

(38) Perdew, J. P.; Ernzerhof, M.; Burke, K. Rationale for mixing exact exchange with density functional approximations. J. Chem. Phys. 1996, 105, 9982-9985.

(39) Weigend, F.; Ahlrichs, R. Balanced basis sets of split valence, triple zeta valence and quadruple zeta valence quality for $\mathrm{H}$ to Rn: Design and assessment of accuracy. Phys. Chem. Chem. Phys. 2005, 7, 3297-3305.

(40) Schäfer, A.; Horn, H.; Ahlrichs, R. Fully Optimized Contracted Gaussian-Basis Sets for Atoms Li to Kr. J. Chem. Phys. 1992, 97, 2571-2577.

(41) Sinnokrot, M. O.; Sherrill, C. D. Highly Accurate Coupled Cluster Potential Energy 
Curves for the Benzene Dimer: Sandwich, T-Shaped, and Parallel-Displaced Configurations. J. Phys. Chem. A 2004, 108, 10200-10207.

(42) Grimme, S.; Antony, J.; Ehrlich, S.; Krieg, H. A consistent and accurate ab initio parametrization of density functional dispersion correction (DFT-D) for the 94 elements H-Pu. J. Chem. Phys. 2010, 132, 154104.

(43) Becke, A. D. Density-functional thermochemistry. III. The role of exact exchange. J. Chem. Phys. 1993, 98, 5648-5652.

(44) Lee, C.; Yang, W.; Parr, R. G. Development of the Colle-Salvetti correlation-energy formula into a functional of the electron density. Phys. Rev. B 1988, 37, 785-789.

(45) Häser, M.; Ahlrichs, R.; Baron, H. P.; Weis, P.; Horn, H. Direct computation of 2ndorder SCF properties of large molecules on workstation computers with an application to large carbon clusters. Theoret. Chim. Acta 1992, 83, 455-470.

(46) Kollwitz, M.; Gauss, J. A direct implementation of the GIAO-MBPT(2) method for calculating NMR chemical shifts. Application to the naphthalenium and anthracenium ions. Chem. Phys. Letters 1996, 260, 639-646.

(47) Jusélius, J.; Sundholm, D.; Gauss, J. Calculation of Current Densities using GaugeIncluding Atomic Orbitals. J. Chem. Phys. 2004, 121, 3952-3963.

(48) Taubert, S.; Sundholm, D.; Jusélius, J. Calculation of spin-current densities using gauge-including atomic orbitals. J. Chem. Phys. 2011, 134, 054123:1-12.

(49) Fliegl, H.; Taubert, S.; Lehtonen, O.; Sundholm, D. The gauge including magnetically induced current method. Phys. Chem. Chem. Phys. 2011, 13, 20500-20518.

(50) Sundholm, D.; Fliegl, H.; Berger, R. J. Calculations of magnetically induced current densities: theory and applications. WIREs Comput. Mol. Sci. 2016, 6, 639-678. 
(51) London, F. Théorie quantique des courants interatomiques dans les combinaisons aromatiques. J. Phys. Radium 1937, 8, 397-409.

(52) Hameka, H. F. On the nuclear magnetic shielding in the hydrogen molecule. Mol. Phys. $\mathbf{1 9 5 8 , ~ 1 , ~ 2 0 3 - 2 1 5 . ~}$

(53) Ditchfield, R. Self-Consistent Perturbation-Theory Of Diamagnetism .1. GaugeInvariant LCAO Method For NMR Chemical-Shifts. Mol. Phys. 1974, 27, 789-807.

(54) Wolinski, K.; Hinton, J. F.; Pulay, P. Efficient implementation of the gauge-independent atomic orbital method for NMR chemical shift calculations. J. Am. Chem. Soc. 1990, 112, 8251-8260.

(55) Jusélius, J.; Sundholm, D. The aromaticity and antiaromaticity of dehydroannulenes. Phys. Chem. Chem. Phys. 2001, 3, 2433-2437.

(56) Pople, J. A.; Untch, K. G. Induced Paramagnetic Ring Currents. J. Am. Chem. Soc. 1966, 88, 4811-4815.

(57) Jusélius, J.; Sundholm, D. Polycyclic Antiaromatic Hydrocarbon. Phys. Chem. Chem. Phys. 2008, 10, 6630-6634.

(58) Singh, U. C.; Kollman, P. A. An approach to computing electrostatic charges for molecules. J. Comp. Chem. 1984, 5, 129-145.

(59) Lazzeretti, P. Ring currents. Prog. Nucl. Magn. Reson. Spectrosc. 2000, 36, 1-88. 
Graphical abstract

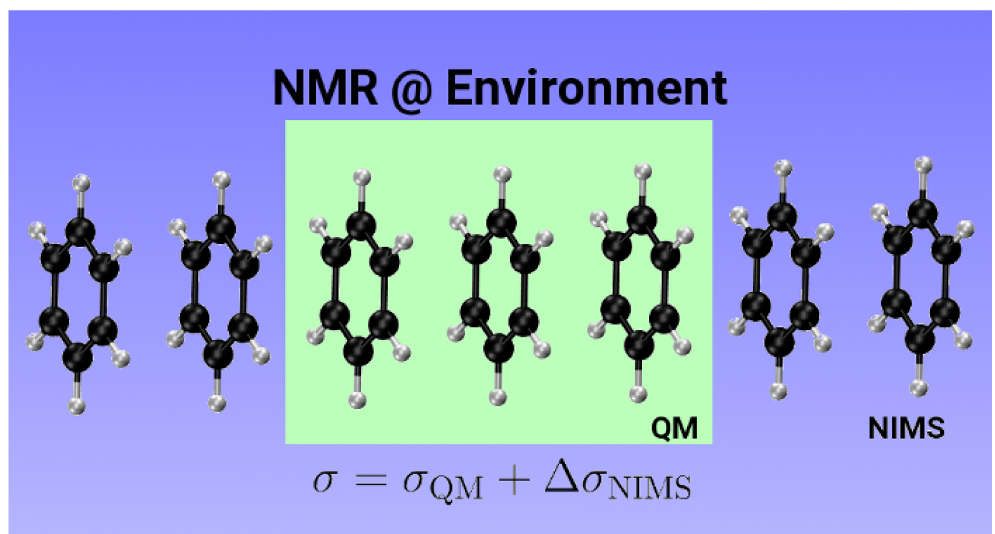

This material is available free of charge via the Internet at http://pubs.acs.org/. 